\title{
A Probabilistic Approach to the Problem of Large Antenna Arrays
}

\author{
Y. T. Lo \\ Contribution From the Antenna Laboratory, Department of Electrical Engineering, University of Illinois, \\ Urbana, Ill.
}

(Received January 2, 1964)

\begin{abstract}
Antenna arrays of very high resolution can be designed without using a large number of elements if they are spaced properly. However, there exists no general theory which would yield a solution to this problem because of its analytical difficulty. In this paper a probabilistic approach is made despite the problem itself being not probabilistic originally. This approach is tantamount to the study of all outcomes for many possible element positions (under a certain rule) by invoking the well-known powerful law of large numbers in probability.

From this investigation it is found that the sidelobe level is closely related to the number of elements, and to a much lesser degree to the aperture dimension. As a result extremely high resolution could be achieved with very few elements. On the other hand, for a given number of elements higher and higher resolution could be obtained by spreading these elements over a larger and larger aperture whereas the sidelobe level would remain substantially the same and the directive gain constant.

Four sample arrays with 100 to 1000 elements have been designed by the Monte Carlo method. Statistical properties of the computed patterns agree almost exactly with those predicted from the theory. Their sidelobe levels also agree with the theoretical values remarkably closely.

For practical interest it is shown that an array can be actually designed to produce a beamwidth of about 1 minute of are by using only 100 isotropic elements over an aperture of $4 \times 10^{3}$ wavelengths, the sidelobe level being $-8.4 \mathrm{~dB}$ and the directive gain $20 \mathrm{~dB}$ over that of a single element. For the sample array of 1000 elements, the beamwidth is 0.5 minutes of are with sidelobe level of $-18 \mathrm{~dB}$. It is also possible to reduce this beamwidth appreciably.
\end{abstract}

\section{Introduction}

Recent advances in space exploration have shown a great need for antennas with high resolution, high gain, and low sidelobe level. Steerable reflector antennas of large size are generally considered to be prohibitive in cost, whereas fixed reflector antennas have a limited range of scanning capability. Hence, attention has turned to large phased arrays. Arrays with uniformly spaced elements have been studied in great detail in the past. It is well known that since the pattern functions of these arrays are periodic in the reduced angular variable of observation, to produce a single beam in the visible range the required number of elements, being in direct proportion to the aperture dimension, is very large. (For example a linear array with an aperture of $5 \times 10^{3}$ wavelengths to produce a beamwidth of 1 min of arc would require $10^{4}$ elements.)

Arrays with incommensurable element spacings usually have aperiodic pattern functions. As a result, the number of elements required is not directly determined by the aperture dimension. This fact has been discovered by a number of antenna workers [King, Packard, and Thomas, 1960; Swenson and Lo, 1961; Andreason, 1962; Maffett; 1962; Ishimaru, 1962; Yen, and Chow 1963]. Some of these works are devoted to uncovering the properties of nonuniformly spaced arrays by studying, a few particular examples with a computer, whereas others are intended for estimating the pattern functions of those arrays whose element positions are determined by certain functions with the aid of some mathematical tables. At present there is no general theory available for this class of arrays.

This paper is a sequel to a previous one [ [ 1964] where a probabilistic approach is proposed, in spite of the fact that this problem itself is not probabilistic originally. ${ }^{1}$ More specifically, arrays with

1 It appears that such an approach has not been well understood by some an. tenna workers who are not familiar with the modern probability theory. Today, probability is regarded as a branch of measure theory by which the probability has been made rigorous, without relying on vague physical intuition. For example, independence can be defined on the properties of measure. In fact, example, independence can be defined on the properties of meastre. In fact, the normal law of large numbers has been suggested by $\mathrm{M}$ arkov and rigorously proven by I.evy without using the physical notion of randomness. It simply states the asymptotical behavior of a quantity under a quite general condition. All of these can be found in a stimulating monoraph entitled, "Statistical Independence in Frobability, Analysis and Number Theory," by M. Kac. oncilable as they may appear at first sight. He has ingeniously demonstrated what probability can do about many deterministic problems such as those in what probability calgebraic equations, continuous fractions, etc. In recent years number theory, algebraic equations, cound particularly impressive as seen from the application to number theory is found particularly impressive as and Ju. V. I. P. Kubiljus, "Probability Methods in Number Theory" 1959], and Ju. I Linnik, "The Dispersion Method in Binary Additive Problems" [961]. In the opening paragraph of the preface in Linnik's book, he asserts that . . such an approach (probabilistic) permits the solution of several binary additive problems which are inaccescible to the known methods ...". This clearly state the motivation of the probabilistic approach to the antenna problem discussed in this paner. 
elements placed at random are considered and their probabilistic properties are studied. The purpose is to determine the condition under which the number of elements could be reduced. It is found that this number is mainly determined by the desired sidelobe level. In this paper the application of this theory will be emphasized. For example, a beamwidth of $1.2 \mathrm{~min}$ of arc could be achieved with only 100 uniformly excited elements over an aperture of $4 \times 10^{3}$ wavelengths; the sidelobe level would be $-8 \mathrm{~dB}$ and the gain $20 \mathrm{~dB}$ over that of a single element. This somewhat surprising result as well as a few others can be predicted from the theory and are subsequently verified by the Monte Carlo experiments in this paper.

The major part of the theory has been established and reported elsewhere [Lo, 1962b]. However, for completeness only a few main results will be first summarized here. Then some experimental results and a few statistics of antenna characteristics will be discussed and compared with the theoretical predictions. This will also illustrate how the theory is applied.

It is perhaps of interest to point out that this problem is related to Weyl's sum in number theory [Vinogradov, 1954] and to the random series studied by Steinhaus [1929]. There is also a similarity between this problem and waves scattered by randomly distributed simple objects, except that in this case the contributions from all elements are coherent in a certain direction in space and also the outcome of the random events could be controlled to a certain extent. Indeed in this approach the design of an array is reduced to playing a game of chance, which could be so designed that the odds would be overwhelmingly in favor of success.

\section{Statement of the Problem}

For simplicity a linear array with uniformly excited elements will be considered. Let the number of elements be $N$ and also let the array with a normalized aperture $[-1,1]$ lie along the $X$ axis of a Cartesian coordinate system. Denote $X_{n}$ for the position of the $n$th element and assume the set of random variables $\left\{X_{n}\right\}$ be independent with a common probability density function $g(x)$. Then the normalized pattern function is given by

$$
P(u)=\frac{1}{N} \sum_{n=1}^{N} \exp i u X_{n}
$$

where $u=a \pi(\sin \theta-\sin \alpha)=$ reduced variable of the angle of observation,

$\Theta=$ observation angle measured from the normal to the array axis, namely to $X$-axis,

$\alpha=$ main beam angle also measured from the above normal,

$a=$ actual aperture dimension in wavelengths.
Mathematically speaking, (1) is a mapping of a random vector defined in an $N$-dimensional cube $-1 \leq X_{n} \leq 1$ into a scalar function $P(u)$. By giving: $g(x)$ it is required to determine some probabilistic properties of the function $P(u)$. Those of practical interest are the beamwidth, antenna response at each observation angle, sidelobe level in the visible region, and directive gain.

\section{Distribution of Radiation Characteristics at Each Observation Angle}

Let the real and imaginary parts of $P(u)$ be $P_{1}(u)$ and $P_{2}(u)$, respectively. Then by the central limit theorem the joint distribution of $P_{1}(u)$ and $P_{2}(u)$ is asymptotically normal. Again for simplicity assume $g(x)$ even; then the joint density function at each $u$ is given by (with $u$ deleted):

$$
f\left(P_{1}, P_{2}\right)=\frac{1}{2 \pi \sigma_{1} \sigma_{2}} \exp -\frac{1}{2}\left[\frac{\left(P_{1}-\varphi\right)^{2}}{\sigma_{1}^{2}}+\frac{P_{2}^{2}}{\sigma_{2}^{2}}\right]
$$

where

$$
\begin{aligned}
& \varphi(u)=E\left\{P_{1}(u)\right\}=\int_{-1}^{1} g(x) \exp i u x d x \\
& =\text { characteristic function of } X \\
& E\left\{P_{2}(u)\right\}=0 \\
& \sigma_{1}^{2}(u)=\frac{1}{2 N}[1+\varphi(2 u)]-\frac{1}{N} \varphi^{2}(u) \\
& \sigma_{2}^{2}(u)=\frac{1}{2 N}[1-\varphi(2 u)] .
\end{aligned}
$$

Hence for any $u$ the probability for the antenna response being less than $r$ is

$$
\operatorname{Pr}\{|P(u)|<r\}=\underset{|P(u)|<r}{\int} \mathcal{S}_{<r} f\left(P_{1}, P_{2}\right) d P_{1} d P_{2}
$$

This is a generalized noncentral chi-square distribution with two degrees of freedom. Tables of percentiles of $r$ for various values of parameters have been compiled by DiDonato and Jarnagin [1960]. When $r$ is large as compared with $\sigma_{1}$ and $\sigma_{2}$, an asymptotic expansion can be found (see appendix A). The approximation due to Patnaik [1949] may also be found useful. In his method a generalized noncentral chi-square distribution of any degree of freedom is approximated by a central one with different degrees of freedom, depending: on the noncentral parameter. The latter can be read off from an imcomplete gamma function tabulated by Pearson [1934]. For details see appendix B.

It is important to notice that since $g(x)$ has a finite support, $\varphi(u)$ is an integral transcendental function of the exponential type with exponent $\leq 1$. 
By Lebesque-Riemann theorem, $\varphi(u)$ approaches zero as $u$ approaches infinity on the real axis. In fact if $\tau$ is the order of zero of $g(x)$ at $x= \pm 1$, $|\varphi(u)| \sim|u|^{-1-\tau}$. Thus for almost all cases of practical interest, $\sigma_{1}(u) \approx \sigma_{2}(u) \approx 1 / 2 N$ and $\varphi(u) \approx 0$ as $u$ is outside of the main beam region. As a result the distribution of $|\mathrm{P}(u)|$ for large $u$, becomes simply chi-square and independent of $u$.

\section{Approximate Distribution of Sidelobe Level}

The sidelobe level is defined as the supremum of the random pattern function $|P(u)|$ for $u$ in the visible range $U$ excluding the main beam region. To this author's knowledge, a rigorous method for determining the distribution of this quantity is unknown. By using the fact that $P(u)$ is analytic with a probability 1 , the probability for $|P(u)|<r$ for all $u$ in $U$ excluding the main beam region, say $U_{0}$, can be approximately estimated by [Lo, $1962 \mathrm{~b}$, 1964]

$$
\operatorname{Pr}\left\{|P(u)|<r, u \epsilon U_{0}\right\} \approx\left(1-10^{-0.4343 N r^{2}}\right)^{2[2 \mathrm{a}]},
$$

where [2a] is the larger of the two integers nearest to $2 \mathrm{a}$. In the case of a symmetrical array to be considered later (i.e., one with an element at $-X_{n}$ whenever there is an element at $X_{n}$ ), the above probability measure becomes

$$
\begin{aligned}
\operatorname{Pr}\{|P(u)| & \left.<r, u \epsilon U_{0}\right\} \approx(\operatorname{erf}(\sqrt{N / 2} r))^{[2 a]} \\
& =\left\{1-\frac{e^{-N r^{2} / 2}}{\sqrt{\pi N / 2} r}\left(1-\frac{1}{N r^{2}}+\ldots\right)\right\}^{[2 a]}
\end{aligned}
$$

where erf $(x)$ is the ferror function of $X$ and the expansion applies for large $\sqrt{N} r$.

It is interesting to see that (4) and (5) imply that, for a given probability, $N$ is closely related to the sidelobe level $r$ and to a much lesser degree to the aperture dimension $a$. In fact for a given $r$, there exists a number $N$, called the critical number of elements, below which the probability of achieving the sidelobe level $r$ decreases rapidly to zero and above which the probability increases only slowly. For symmetrical arrays this is shown in figure 1 . A close examination of this chart will show that for a given $N$, say $2 \times 10^{3}$, the sidelobe level would be $-20 \mathrm{~dB}$ for $a=10^{4}$ and $-19.2 \mathrm{~dB}$ for $a=10^{5}$, both with 80 percent probability. Since the beamwidth is inversely proportional to $a$, it is clearly seen that the beamwidth could be reduced by a factor of 10 by increasing $a$ by ten times without significantly raising the sidelobe level. From this result it is also of interest to note that for a given $N$, the sidelobe level is almost fixed, independent of $g(x)$, or a particular set of element positions among many others. The numerical results obtained by many workers cited in section 1 already verified this conclusion.

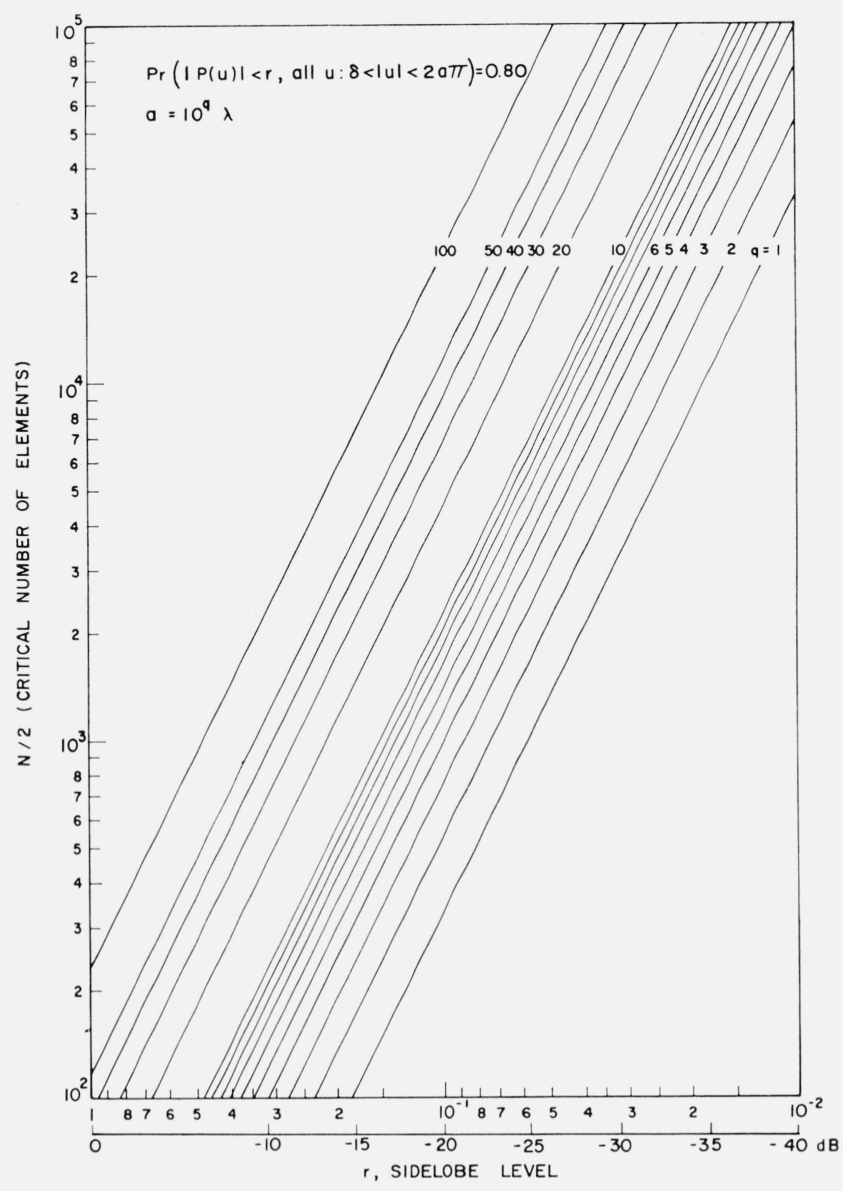

Figure 1. The critical number of elements of a symmetrical array as a function of the sidelobe level with 80 percent probability.

$$
N=\text { total number of elements. }
$$

\section{Distribution of Half-Power Beamwidth}

The random variables $u_{0}$ defined by the two first roots (one positive, the other negative) of the following equation determine the half-power beamwidth completely:

$$
\left|P\left(u_{0}\right)\right|=1 / \sqrt{2}
$$

Using the fact that $P(u)$ is analytical with probability 1 and also the fact that $\sigma_{1}(u)$ and $\sigma_{2}(u)$ are small for small $u$, the random variable $u_{0}$ can be simply and closely approximated by

$$
u_{0} \approx u_{1}-\frac{P_{1}\left(u_{1}\right)-1 / \sqrt{2}}{\varphi^{\prime}\left(u_{1}\right)}
$$

where $u_{1}$ is the corresponding first root of the equation $\varphi\left(u_{1}\right)=1 / \sqrt{2}$, and $\varphi^{\prime}(u)$ is the derivative of $\varphi(u)[$ Lo, 1964]. The above equation implies that, to the first order of approximation $u_{0}$ and $\mathrm{P}_{1}\left(u_{1}\right)$ are linearly related. Since the distribution of $\mathrm{P}_{1}\left(u_{1}\right)$ is known, that of $u_{0}$ can be readily determined. Without going into detail, it is easy to show that for large $a$ and moderately large $N, u_{0}$ is approximately equal to $u_{1}$ with a probability nearly equal to 1 . 


\section{Distribution of the "Distance" and Direc- tive Gain Between the Random Pattern Function $\mathrm{P}(u)$ and the Desired Pat- tern $\varphi(u)$}

An antenna array may be regarded as a finite set of samples taken from a continuous excitation $g(x)$ over the aperture [Lo, 1962a]. In this approach the pattern function of the latter, namely $\varphi(u)$, may be considered as a reference or desired pattern since the technique for designing an antenna with continuous excitation is well known. Thereiore, it is of interest to investigate the difference between $P(u)$ and $\varphi(u)$.

In Hilbert space this difference can be expressed by the "distance" between $P(u)$ and $\varphi(u)$ which is defined to be the norm $\| P-\varphi \mid$. Following a theorem due to Karhunen [1947] and Loève [1960], $P-\varphi$ admits a spectral representation; then the distribution of $\|P-\varphi\|^{2}$ can be determined as given by [Lo, 1962b, 1964]

$\operatorname{Pr}\left\{D<\gamma^{2}\right\}=\Phi_{0}\left[\frac{\gamma^{2}-E\{D\}}{\sigma\{D\}}\right]-\Phi_{0}\left[-\frac{E\{D\}}{\sigma\{D\}}\right]$

where

$$
\begin{aligned}
& D=|P-\varphi|^{2}=\int_{U}|P(u)-\varphi(u)|^{2} d u \\
& E\{D\} \approx 2 \pi d_{\mathrm{av}}-2 \|\left. g\right|^{2} / N \\
& \sigma^{2}\{D\} \approx 8 \pi^{2} d_{\mathrm{av}} \| g||^{2} / N \\
& d_{\mathrm{av}}=a / N, \|\left. g\right|^{2}=\int_{-1}^{1}|g(x)|^{2} d x,
\end{aligned}
$$

$\Phi_{0}(x)=$ the standardized normal distribution function.

From the above it can be shown that the difference between $G_{0}$, the directive gain of the reference antenna pattern $\varphi(u)$, and $G$, that of the random pattern $P(u)$, is given by

$$
\left(G_{0}-G\right) \mathrm{dB} \leq 20 \log \left(1+\left(\sqrt{d_{\mathrm{av}}} / \| g||\right)\right)
$$

with a probability nearly equal to 1 . For large $d_{\text {av }}$ (i.e., when elements are widely spaced) $G$ becomes proportional to $N$.

With reference to the results obtained in the previous sections, the following interesting conclusions can be drawn: For a given $N$, there exists a sidelobe level which is likely to be achieved. This level increases extremely slowly with the aperture dimension $a$. Thus for given $N$ and $a$ the beamwidth could be reduced by a factor of 10 or 100 by merely increasing $a$ to $10 a$ or $100 a$. In so doing the risk of obtaining a much higher sidelobe level is very small. The gain, being proportional to $N$, will remain unchanged. This conclusion has been verified quantitatively by the Monte Carlo experiments, as will be seen in section 8 .

\section{Stationarity and Ergodicity Approximation}

By referring to (1), as $u=0, P(u)$ is deterministic and equal to 1 . For small $u$ and large $N$, the variances are very small quantities and $\digamma(u)$ is nearly deterministic. Therefore, in general, the behavior of $P(u)$ requires attention only when $u$ is outside of the main beam region. Fortunately for large $u, P(u)$ behaves like Gaussian noise with $\sigma_{1}^{2} \approx \sigma_{2}^{2} \approx 1 / 2 N$ and $\mathrm{E}\{F(u)\} \approx 0$. Thus following Woodward [1953] one may speak of "optimum" pattern in the sense that it has a maximum entropy; i.e., statistically speaking all sidelobes have equal level with equal probabilities. Also for large $u, P(u)$ becomes stationary, since by a simple mathematical manipulation its covariance function is found to be

$$
K(u, v)=\frac{1}{N}\left[\varphi(u-v)-\varphi(u) \varphi^{*}(v)\right] \approx \frac{1}{N} \varphi(u-v)
$$

for large $u$ and $v$, where $*$ implies the complex conjugate and the last approximation is due to the asymptotic behavior of $\varphi(u)$ as discussed in section 3. Since $P(u)$ is asymptotically normal and its spectral distribution function, in general, is continuous, by Maruyama's theorem [1949] $P(u)$ is metrically transitive, hence ergodic for sufficiently large $N$ and $u$. It follows that the "time" average over $u$ is equal to the "space" average over $\omega$ in probability space. As a consequence, for large $u$, the statistical behavior of a sample function $P_{\omega}(u)$ observed over a long "time" interval $U_{0}$ can be approximately determined from the probability distribution at a fixed $u$, which is simply given by (2). In a few examples studied it is found that the general behavior of the sample function $\left|P_{\omega}(u)\right|$ can be predicted from this theory with high accuracy, as will be seen in the next section.

\section{Statistics of a Few Sample Arrays}

Four symmetrical arrays with $100,300,600$, and 1000 elements, respectively, have been designed by the Monte Carlo method, according to a scheme implied in the theory. It is remarkable to find that in just a single trial for each case the statistics of various antenna properties showed a close agreement between the theory and experiment.

The Monte Carlo Method is a random experiment simulated by a computer, involving the following essential steps. First a set of random numbers with uniform distribution must be generated. Various methods are available. For example, a million random digits published by the Rand Corporation were generated by a chance-dependent electronic device. Since von Neumann introduced the midsquare method, the analytical approach becomes more widely used today. The numbers so obtained are generally referred to as the pseudorandom numbers since they are generated by a deterministic method. In recent years with the aid of modern 
computers, pseudorandom numbers can be easily generated by various congruential methods [Greenberger, 1961]. Then from these numbers, samples of a random variable of any distribution can be obtained by the probability integral transformation.

In this paper, in order to show how simply one can determine the element positions, 500 random numbers have been drawn directly from Owen's table [1962]. The poker test and the chi-square goodnessof-fit tests for the frequencies of digits, pairs, and distance between zeros of these numbers have been given by Owen and are shown below:

\begin{tabular}{c|c|c|c}
\hline Poker & $\begin{array}{c}\text { Frequency of } \\
\text { digits }\end{array}$ & $\begin{array}{c}\text { Frequency of } \\
\text { pairs }\end{array}$ & $\begin{array}{c}\text { Distance be- } \\
\text { tween zeros }\end{array}$ \\
\hline 1.0 & 6.9 & 89.3 & 17.0 \\
\hline
\end{tabular}

Again assume $g(x)=\cos ^{2} \pi x / 2$; then by probability integral transformation the corresponding samples for this density function can be obtained. The distributions of these samples are shown in figure 2 along with the theoretical probability distribution function. However, to avoid unnecessary complications only positions of every fifth element are shown with dots in this figure.

The pattern functions $|P(u)|$ for symmetrical arrays of $100,300,600$, and 1000 elements with their positions determined respectively by the first 50, 150,300 , and 500 random numbers of Owen's table cited above have been computed by IBM Computer 7094. To assure that the largest sidelobe maximum will be obtained the pattern function for each case is computed at $4 \times 10^{4}$ equispaced points for $u / \pi$ in a range from 0 to $10^{4}$ (corresponding to $a=10^{4}$ ). For $u / \pi$ in the interval $(0,19)$, they are plotted in figure 3 .

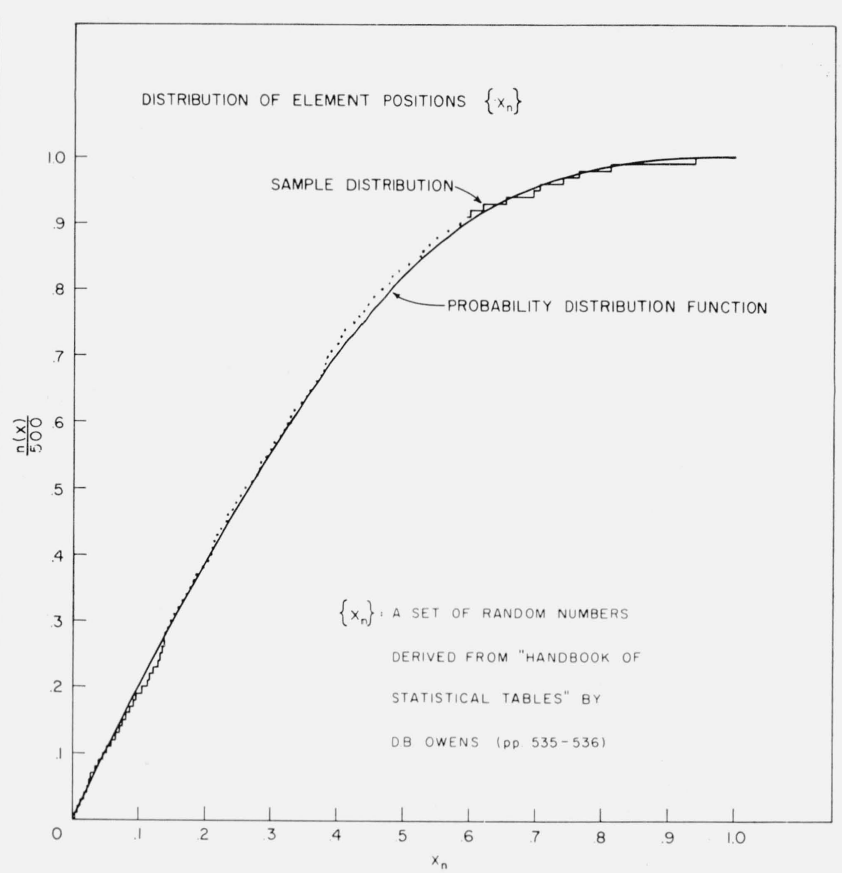

Figure 2. Distribution of element positions. ... . Sample distribution, $-\_$population distribution

It is seen that regardless of the number of elements all have the same half-power beamwidth and nearly identical main beam as expected from the theory in section 5. As for the rest, a pattern plot becomes not only impractical, but also of little importance. Therefore a statistical study of $P(u)$ has been made.

Since for a symmetrical array $P_{2}(u)=0$, it follows from (2) that for each $u, P(u)$ has a normal distribution. On the other hand, in the last section it has been established that for large $u$ and $N, P(u)$ is approximately ergodic. Therefore, it is of great interest to compare the distribution of actually com-

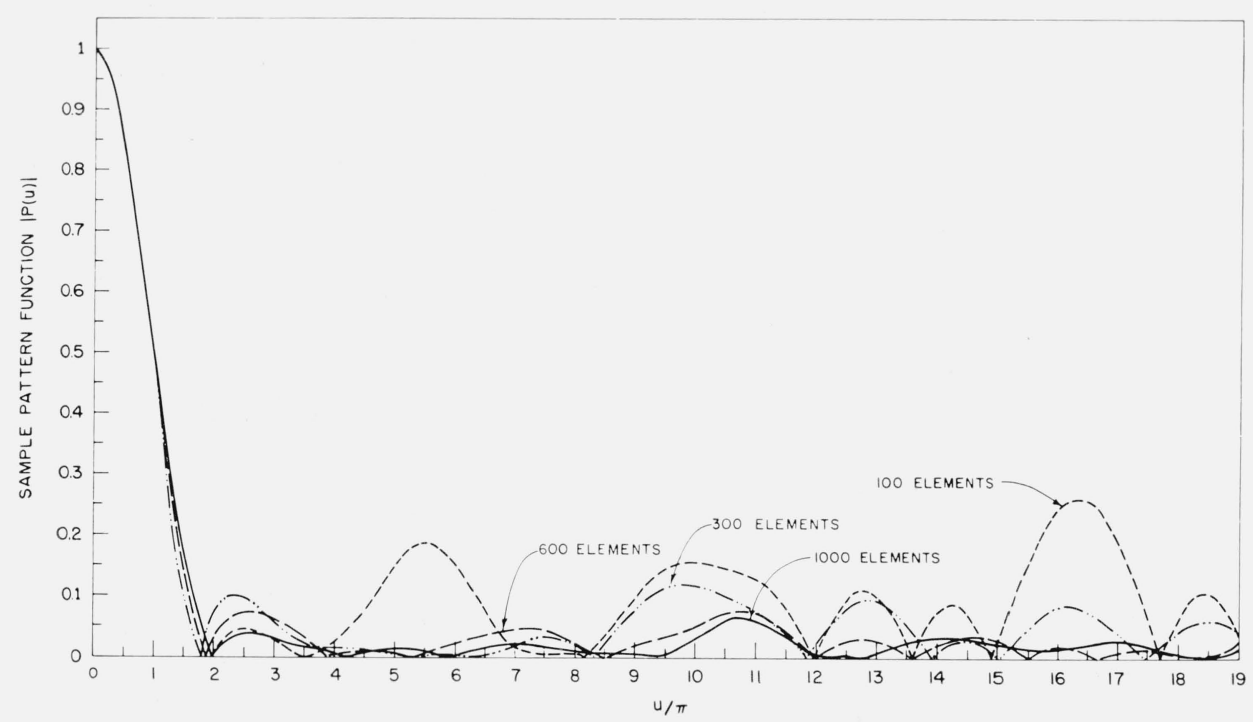

Figure 3. Sample pattern functions of symmetrical arrays with $\mathrm{N}=100,300,600$, and 1000 elements, respectively. 
puted values of $P(u)$ (excluding those for small $u$ 's) with a normal curve. In the case of $10^{3}$ elements this is shown by a histogram in figure 4 . The middle points of each digitized step lie close to the normal curve. Since actually $|P(u)|$ is of more interest in practice, a histogram of $(P / \sigma)^{2}$ is plotted along with the $\chi^{2}$-distribution density function in figure 5 . In this case the midpoints of all steps lie almost exactly on the theoretical curve. Figure 6 shows the sample distribution function and the $\chi^{2}$-distribution. It is seen that they agree very closely. These results have verified the ergodicity approximation obtained earlier. In application the converse is important; namely, from the theoretical curves one can predict the frequency distribution of the antenna responses over all angles with extremely high accuracy.

Long "time" correlation functions of the above sample pattern for $u$ in various ranges, each averaged over a length equal to $10^{3} \pi$, have also been computed. When $|u-v| / \pi<2$, they agree closely with the covariance function given by (9). For $|u-v|$ outside this interval, the correlation function becomes random-like. A typical curve averaged over $u$ from $5 \pi \times 10^{3}$ to $6 \pi \times 10^{3}$ is shown in figure 7 . It is believed that the disagreement of this curve with (9) for $|u-v| / \pi\rangle 2$ is due to the insufficient length of $u$ used for averaging. However the details in this portion of the curve are of minor importance.

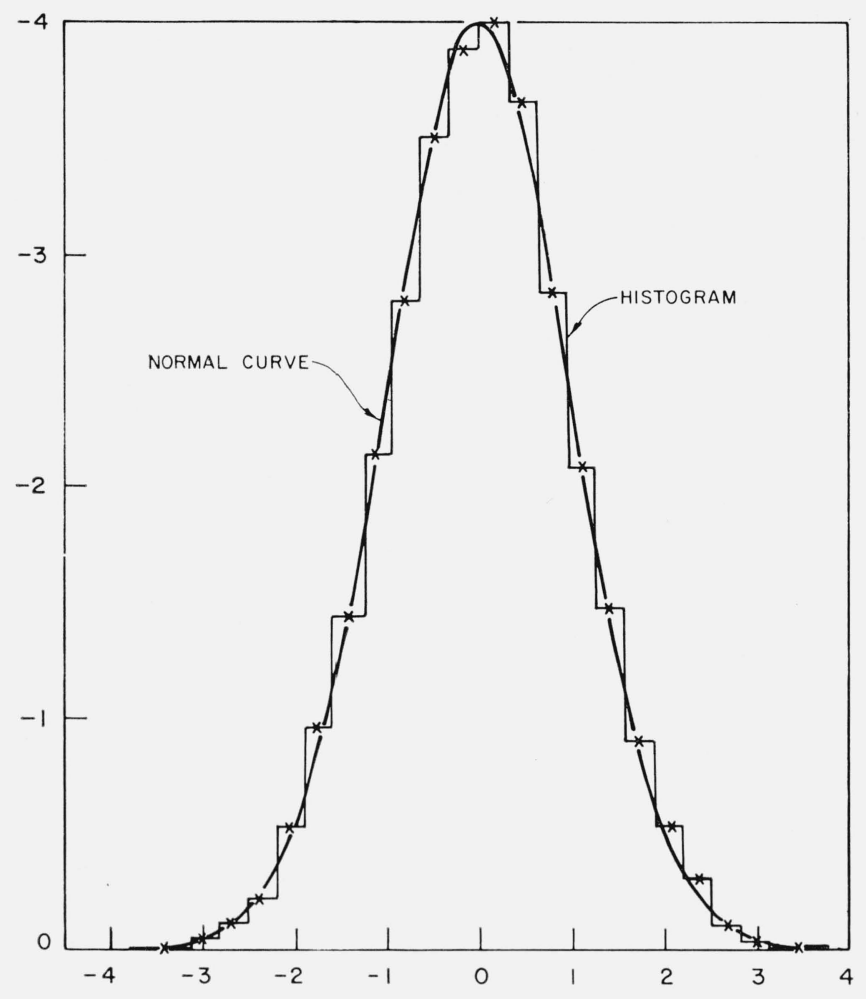

$(P / \sigma)$

Figure 4. Histogram of the normalized sample pattern function $\mathrm{P} / \sigma$ for $\mathrm{N}=1000$ as compared with the theoretical density Junction, namely the normal curve.

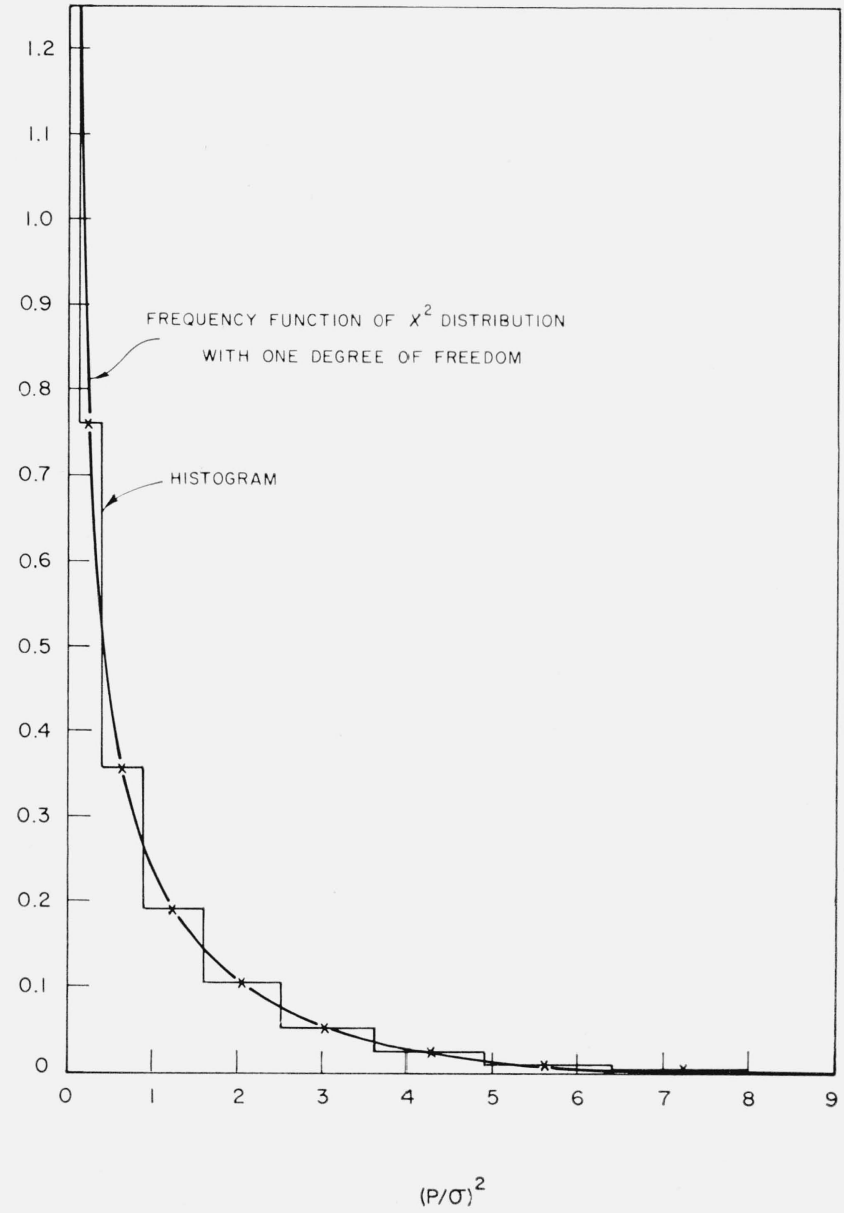

Figure 5. Histogram of the square of the normalized pattern function $(\mathrm{P} / \sigma)^{2}$ for $\mathrm{N}=1000$ as compared with the theoretical density function, namely the chi-square density function with $1 \mathrm{deg}$ of freedom.

Finally figure 8 shows the most important feature of these arrays, namely the sidelobe level, where the theoretical curves are obtained from figure 1 . Since in this paper normalized aperture is used (see (1)), a change in the actual aperture dimension corresponds to a proportional change in $u$, and hence a corresponding change in beamwidth. This is indicated by the half-power beamwidth scale in minutes of arc in the figure.

The agreement between the experimental and the theoretically predicted results, though not as close as other properties studied earlier, is considered quite remarkable, particularly in view of a few methods suggested by other authors [Lo, 1963]. In addition, the present method is both extremely simple and general. As stated earlier the chart in figure 1 is more accurate for large $N$. This is also seen in this figure.

From these results it is interesting to find that an array can be designed to produce a beam of about 1 min of arc with only 100 isotropic elements over an aperture on the order of $4 \times 10^{3} \lambda$. The sidelobe level would be $-8.4 \mathrm{~dB}$ and directive gain would be $20 \mathrm{~dB}$ over a single element. In case of a conven- 
tional design with uniformly spaced, elements at least $4 \times 10^{3}$ of them would be required.

Of course, the gain for the latter will be substantially higher-roughly by a factor of 40 in power ratio if they are uniformly excited. In that case it is wellknown that the sidelobe level will be $-13 \mathrm{~dB}$. On the other hand if these $4 \times 10^{3}$ elements were spread at random over a larger aperture, say $4 \times 10^{5} \lambda$, a much narrower beam, about one-hundredth of that of the uniformly spaced array, could be obtained with practically no loss in gain. In addition, by referring to figure 1 , the sidelobe level would be considerably lower, about $-21 \mathrm{~dB}$.

When $N=300$ and the aperture equals $5 \times 10^{3} \lambda$, the half-power beamwidth will be about $1 \mathrm{~min}$ of are. The sidelobe level as found from figure 8 is equal to $-13 \mathrm{~dB}$, about the same as that of the uniformly spaced counterpart, but the number of elements is only 6 percent of the latter.

Figure 8 also shows that for $10^{3}$ elements and an aperture of $10^{4} \lambda$, a beam of $0.5 \mathrm{~min}$ of arc can be obtained with $-18 \mathrm{~dB}$ sidelobe level. Although the computation was performed only for $u$ up to $10^{4} \pi$, it is expected that the sidelobe would remain at substantially the same level for $u$ increasing to several times this range.

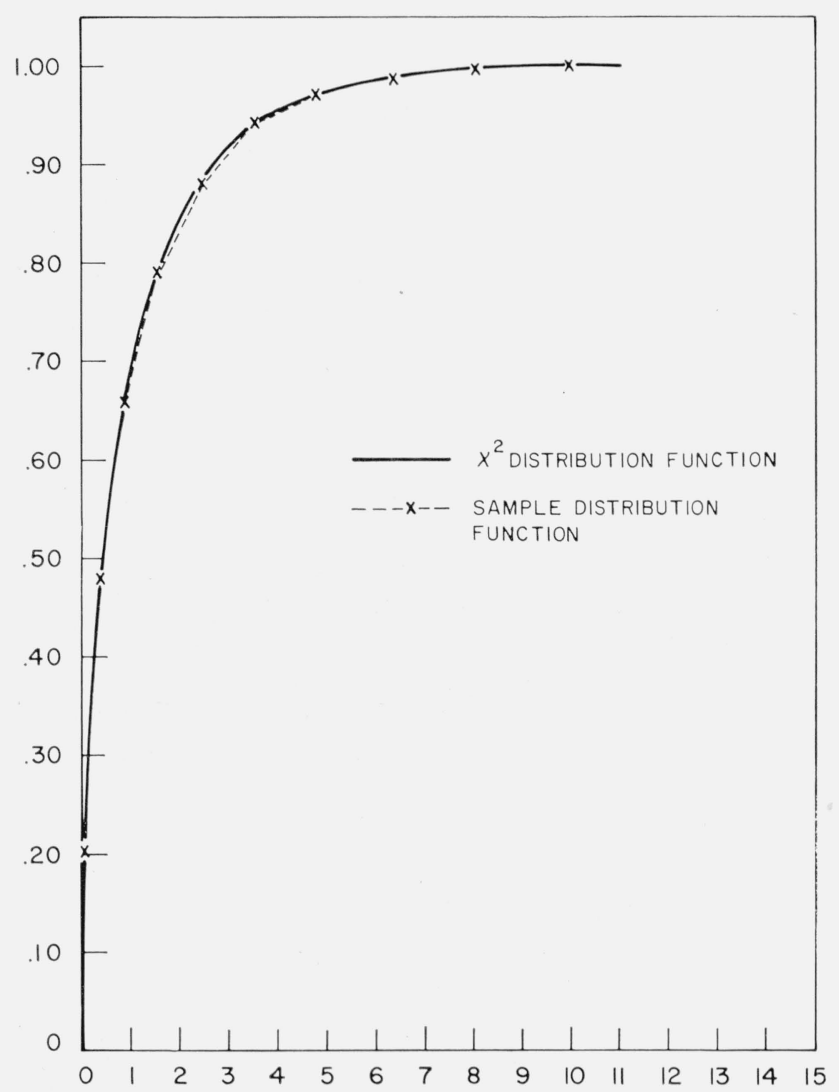

$(P / \sigma)^{2}$

Figure 6. Sample distribution function of $(\mathrm{P} / \sigma)^{2}$ for $\mathrm{N}=1000$ as compared with the theoretical chi-square distribution.

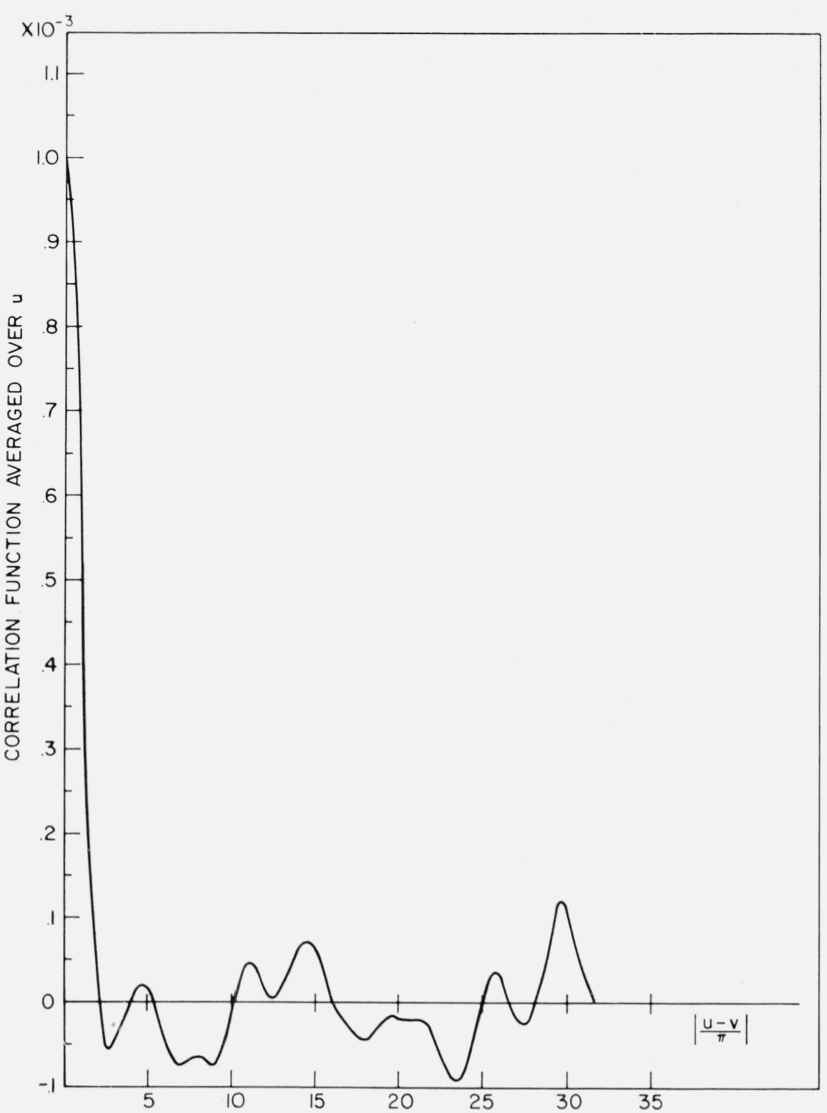

Figure 7. Correlation function of a sample pattern function averaged over the interval $5 \pi \times 10^{3} \leq u \leq 6 \pi \times 10^{3}$.

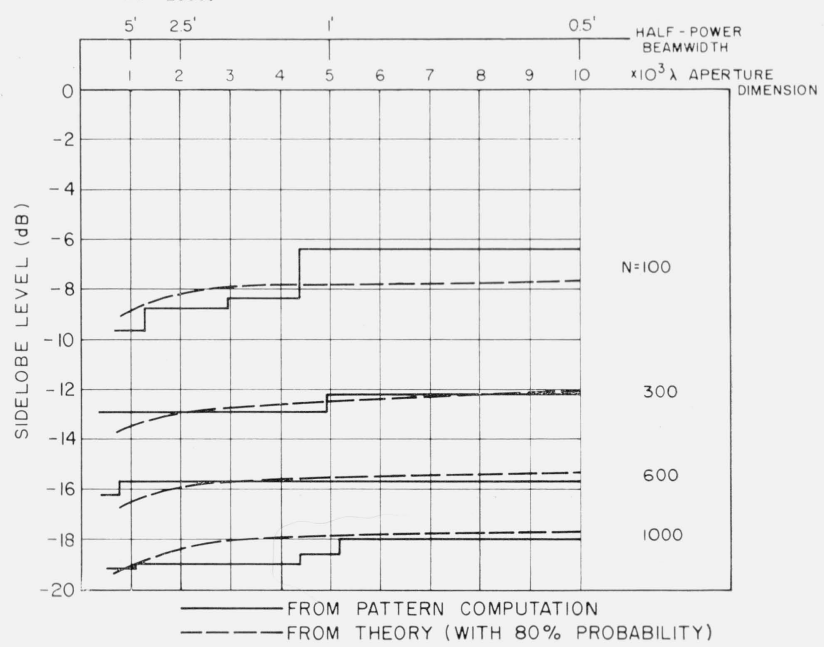

Figure 8. Sidelobe levels of four sample arrays with $N=100$, 300, 600, and 1000 elements as a function of aperture dimensions in wavelengths or half-power beamwidths in minutes of arc.

\section{Conclusion and Remarks}

In this paper the probability theory is used to obtain $a$ solution to a problem which, although not probabilistic origitally, is mathematically intractable otherwise. In this respect, the present approach seems to be similar to the congruential technique 
used in generating pseudorandom numbers, but in a reversed manner. This may become clearer if attention is paid to the fact that the contribution from each term (or element) of (1) is determined by its phase angle $X_{n} u(\bmod 2 \pi)$. In general, even though $\left\{X_{n}\right\}$ may not be random, $\left\{X_{n} u(\bmod 2 \pi)\right\}$ appears like random. Thus the results obtained in this paper may also be applied to many nonuniformly spaced arrays [Lo, 1963].

In essence, this investigation has shown that high resolution can be achieved without the need of a great number of elements unless very high gain is of primary importance. On the other hand, for a given number of elements higher and higher resolution can be obtained by spreading the elements at random over a larger and larger aperture whereas the sidelobe level would remain essentially the same and the directive gain constant. This investigation has also shown that almost all the properties of radiation pattern function can be accurately predicted from the theory without resorting to a high speed computer.

These results are also applicable to arrays of higher dimensions with little modification. In fact, the advantage of using random spacings in these arrays will be even greater.

High order solutions of the distribution function $\operatorname{Pr}\{|P|<r\}$ as shown in (3) have also been obtained by following a method due to Edgeworth who expanded a one-dimensional distribution function in te $m_{s}$ of Hermite-Chebyshev polynomials. However, for the two-dimensional distribution, as in the present case, the expansion becomes too complicated to be presented here. For interested readers, these solutions and their effect on the results in (4) and (5) can be found in Lo [1962b]. However, a rigorous solution to the distribution of the supremum of a random function as in (4) and (5) is still an outstanding problem.

The author acknowledges the support of the $\mathrm{Na}$ tional Science Foundation in this investigation. He also expresses his gratitude to Professor G. A. Deschamps for many interesting discussions, and to Dr. V. Gylys for programming some pattern computations.

\section{Appendix $\bar{A}$}

In this appendix an asymptotic expansion of the generalized noncentral chi-square distribution of two degrees of freedom given by (3) will be determined. This expansion is applicable to the mainbeam region where $r>>\sigma_{1}$ and $\sigma_{2}$. The method used here is to replace the probability measure inside a circle of radius $r$ as shown in (3) by that inside a square region of side equal to $r$ and some error terms. This error, being the probability measure in the region bounded between the circle and the square, is small for large $r$ and can be evaluated asymptotically.

Let $x=P_{1} / \sigma_{1}, \quad y=P_{2} / \sigma_{1}, \quad m=\varphi / \sigma_{1}, \quad s=\sigma_{1} / \sigma_{2}$ then (3) becomes

$$
\begin{array}{r}
\operatorname{Pr}\left\{\sqrt{x^{2}+y^{2}}<r\right\}=\frac{2 s}{\pi} \int_{0}^{r} \int_{-\sqrt{r^{2}-y^{2}}}^{\sqrt{r^{2}+y^{2}}} \\
\exp \left[-(x-m)^{2}-s^{2} y^{2}\right] d x d y \\
=\frac{2 s}{\pi} \int_{0}^{r}\left[\int_{-r}^{r}-\int_{-r}^{--\sqrt{r^{2}-y^{2}}}-\int_{\sqrt{r^{2}-y^{2}}}^{r}\right] \\
\exp \left[-(x-m)^{2}-s^{2} y^{2}\right] d x d y .
\end{array}
$$

The first double integral representing the probability mass in the square region, can be easily evaluated in terms of error function; whereas the second and third double integrals are the error. Now consider the third one and separate the range $y=0$ to $r$ into $y=0$ to $k r$ and $k r$ to $r$, with $0<k<1$; i.e.,

$$
\begin{aligned}
& \frac{2 s}{\pi} \int_{0}^{r} \int_{\sqrt{r^{2}-y^{2}}}^{r} \exp \left[-(x-m)^{2}-s^{2} y^{2}\right] d x d y \\
& \quad=\frac{2 s}{\pi}\left[\int_{0}^{k r}+\int_{k r}^{r}\right] \int_{\sqrt{r^{2}-y^{2}}}^{r} \exp \left[-(x-m)^{2}-s^{2} y^{2}\right] d x d y .
\end{aligned}
$$

Let $\delta=r-m, x^{1}=x-r, y^{1}=y / r . \quad$ First expand the integral into a Taylor series in $x^{1}$ and integrate term by term with respect to $x^{1}$; next expand $\left(\sqrt{r^{2}-y^{2}}-r\right)$ into a power series in $y^{1}$ and integrate again with respect to $y^{1}$. Then one finds that the above double integral reduces to

$$
\begin{aligned}
& \frac{1}{2 \pi s^{2} r} \exp -\delta\left\{\Gamma(3 / 2)+\frac{\delta}{2} \frac{\Gamma(5 / 2)}{s^{2} r}+\left[\frac{\Gamma(5 / 2)}{4 s^{2}}\right.\right. \\
& \left.\left.+\frac{2 \delta^{2}-1}{12 s^{2}} \Gamma(7 / 2)\right] \frac{1}{r^{2}}+0\left(s^{-4} r^{-3}\right)+0\left(\sqrt{s k r} \exp -s^{2} k^{2} r^{2}\right)\right\}
\end{aligned}
$$

Similarly one obtains an asymptotic expansion for the second double integral. By combining these results one has

$$
\begin{array}{r}
\operatorname{Pr}\left\{\sqrt{x^{2}+y^{2}}<r\right\}=\frac{1}{2} \operatorname{erf}(s r)[\operatorname{erf}(r+m)+\operatorname{erf}(r-m)] \\
-\frac{1}{4 \sqrt{\pi} s^{2} r} \exp -\delta^{2}\left\{1+\frac{3 \delta}{4 s^{2} r}+\left[\frac{3}{8 s^{2}}+\frac{15\left(2 \delta^{2}-1\right)}{48 s^{4}}\right] \frac{1}{r^{2}}\right. \\
\left.+0\left(s^{-4} r^{-3}\right)+0\left(\sqrt{s k r} \exp -s^{2} k^{2} r^{2}\right)\right\} .
\end{array}
$$

For the special case when $\sigma_{1}=\sigma_{2}$ (i.e., $s=1$ ) the above expansion reduces to that given by Rice [1945], who utilized the asymptotic expansion of Bessel function in his derivation.

\section{Appendix B}

Following Patnaik [1949], a generalized noncentral chi-square distribution can be approximately computed by a central one with a different parameter and different degrees of freedom. The latter then can be read from Pearson's Table [1934] of incomplete gamma function $I(v, p)$. By following the same notation as in (3): 
where

$$
\operatorname{Pr}\left\{\sqrt{P_{1}^{2}+P_{2}^{2}}<r\right\}=I(v, p)
$$

$$
\begin{aligned}
v & =\frac{r^{2}}{\sqrt{\mu_{2}}}, \quad p=\frac{\mu_{1}^{2}}{\mu_{2}}-1, \\
\mu_{1} & =\sigma_{1}^{2}+\sigma_{2}^{2}+\varphi^{2}, \\
\mu_{2} & =2\left(\sigma_{1}^{4}+\sigma_{2}^{4}+2 \varphi^{2} \sigma_{1}^{2}\right) .
\end{aligned}
$$

For convenience $\mathrm{I}(v, p)$ is plotted against $v$ for various values of $p$ in figure 9 . At $p=0$, it is simply the Rayleigh distribution.

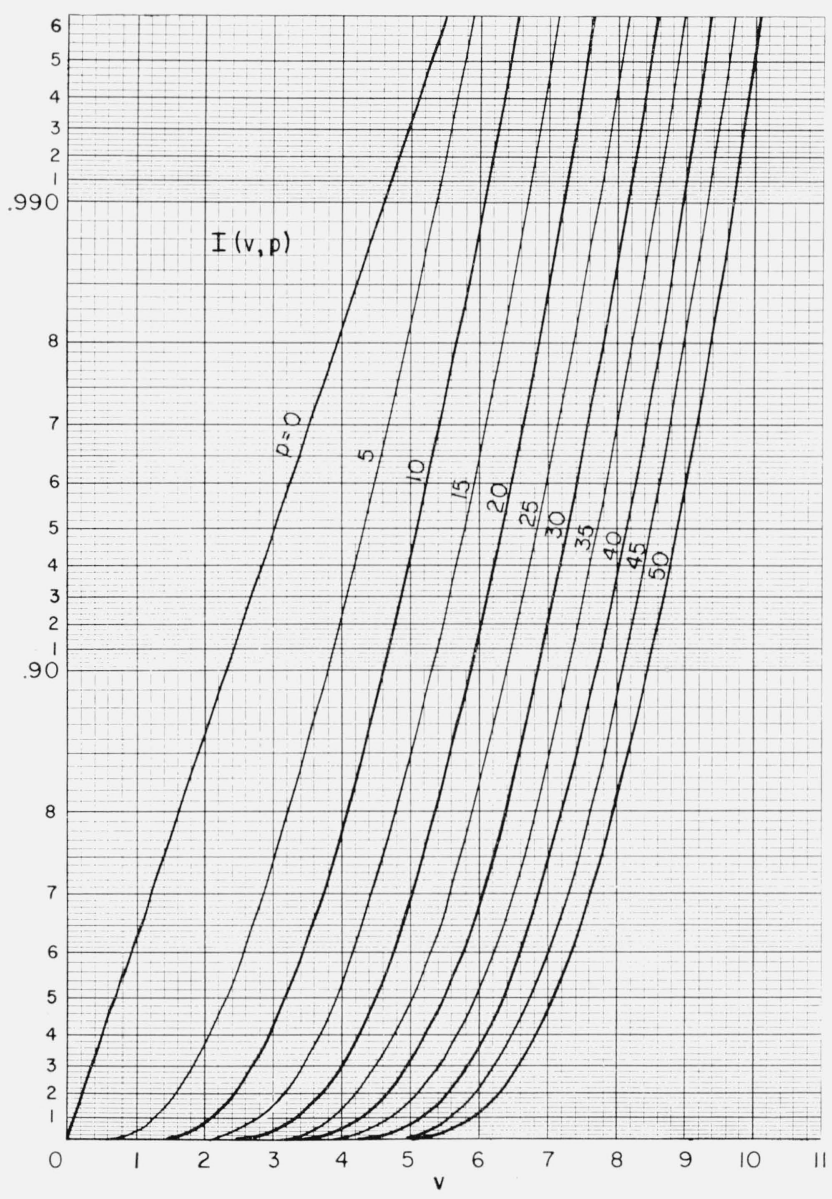

FiguRE 9. Incomplete gamma function $\mathrm{I}(\mathrm{v}, \mathrm{p})$ against $\mathrm{v}$ with $\mathrm{p}$ as parameter.

\section{References}

Andreason, M. G. (1962), Linear arrays with variable interelement spacings, IRE Trans. Ant. Prop., AP-10, 137-143.

DiDonato, A. R., and M. P. Jarnagin (1960), Integration of the general bivarite gaussian distribution over an offset ellipse, NWL Report 1710, U.S. Naval Weapons Laboratory, Dahlgren, Va.

Greenberger, M. (1961), A priori determination of serial correlation in computer generated random numbers, Math. Comp. XV, No. 76, 383-389.

Ishimaru, A. (1962), Theory of unequally spaced arrays, IRE Trans. Ant. Prop. AP-10, 691.

Karhunen, K. (1947), Über linear Methoden in der Wahrscheinlichkeits-Rechnueng, Ann. Acad. Sci. Fennicae, Ser. A, Pt. 1, Math. Phys. 3\%, 79.

King, D. D., R. F. Packard, and R. K. Thomas (1960), Unequally spaced, broadband antenna arrays, IRE Trans. Ant. Prop. AP-8, 380-384.

Lo, Y. T. (1962a), A spacing weighted antenna array, IRE Intern Conv. Record, Pt. 1, 191.

Lo, Y. T. (1962b), On the theory of randomly spaced antenna arrays, Techn. Report sponsored by the National Science Foundation (G14894), Antenna Laboratory, Department of Electrical Engineering, University of Illinois, Urbana, Ill.

Lo, Y. T. (1963), Sidelobe level in nonuniformly spaced antenna arrays, IEEE Trans. Ant. Prop. AP-11, 511-512.

Lo, Y. T. (1964), A mathematical theory of antenna arrays with randomly spaced elements, IEEE Trans. Ant. Prop. AP-12, 257-268.

Loève, M. (1960), Probability Theory, ch. X (D. Van Nostrand Co., New York, N.Y.).

Maffett, A. L. (1962), Array factors with nonuniform spacing parameters, IRE Trans. Ant. Prop. AP-10, 131-136.

Maruyama, G. (1949), The harmonic analysis of stationary stochastic processes, Mem. Fac. Sci. Kyushu University, A4, 45-106.

Owen, D. B, (1962), Handbook of Statistical Tables, pp. 535-536 (Addison-Wesley Pub. Co., Inc., Reading, Mass.).

Patnaik, P. B. (1949), The non-Central $\chi^{2}$ and $F$-distributions and their application, Biometrica 36, 202-232.

Pearson, K. (1934), Tables of incomplete gamma functions, Department of Scientific and Industrial Research,

Steinhaus, H. (1929), Úber die Wehrscheinlichkeit dafur dass der Konvergenzenzkreis einen Potenzreihe ihre natur liche grenze ist, M. Z. 21, 408.

Swenson, G., and Y. T. Lo (1961), The University of Illinois Radio Telescope, IRE Trans. Ant. Prop. AP-9, 9.

Vinogradov, I. M. (1954), The Method of Trigonometerical Sums in the Theory Numbers (Interscience Pub. Co., New York, N.Y.).

Woodward, P. M. (1953), Probability and Information Theory, with application to radar, pp. 25 (Pergamon Press Ltd., London).

Yen, J. L., Y. L. Chow (1963), On large nonuniformly spaced arrays, Can. J. Phys. 41, 1. 